\title{
Incorporation of Host Complement Regulatory Proteins into Newcastle Disease Virus Enhances Complement Evasion
}

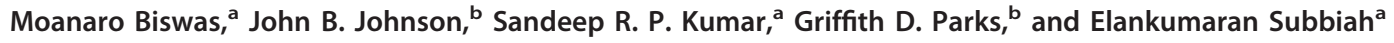 \\ Department of Biomedical Sciences and Pathobiology, Virginia-Maryland Regional College of Veterinary Medicine, Virginia Polytechnic Institute and State University \\ Blacksburg, Virginia, USA, and Department of Microbiology and Immunology, Wake Forest University School of Medicine, Winston-Salem, North Carolina, USA
}

Newcastle disease virus (NDV), an avian paramyxovirus, is inherently tumor selective and is currently being considered as a clinical oncolytic virus and vaccine vector. In this study, we analyzed the effect of complement on the neutralization of NDV purified from embryonated chicken eggs, a common source for virus production. Fresh normal human serum (NHS) neutralized NDV by multiple pathways of complement activation, independent of neutralizing antibodies. Neutralization was associated with $\mathrm{C} 3$ deposition and the activation of C2, C3, C4, and C5 components. Interestingly, NDV grown in mammalian cell lines was resistant to complement neutralization by NHS. To confirm whether the incorporation of regulators of complement activity (RCA) into the viral envelope afforded complement resistance, we grew NDV in CHO cells stably transfected with CD46 or HeLa cells, which strongly express CD46 and CD55. NDV grown in RCA-expressing cells was resistant to complement by incorporating CD46 and CD55 on virions. Mammalian CD46 and CD55 molecules on virions exhibited homologous restriction, since chicken sera devoid of neutralizing antibodies to NDV were able to effectively neutralize these virions. The incorporation of chicken RCA into NDV produced in embryonated eggs similarly provided species specificity toward chicken sera.

Thesing he complement system is a group of more than 20 serum proteins that are activated upon foreign antigen exposure to initiate a rapid, localized, and tightly regulated enzymatic cascade, functioning as an early innate immune response against pathogens, including viruses. Complement may interfere with the initial stages of virus infection through several mechanisms, including steric hindrance of viral receptors to prevent attachment, fusion, and entry into host cells, opsonization by $\mathrm{C} 3 \mathrm{~b}$ and/or C4b proteolytic fragments to induce aggregation, lysis, and clearance by phagocytic cells either in the presence or absence of antibodies and subsequent generation of inflammatory and adaptive immune responses (2).

Inappropriate activation of complement can be potentially damaging to normal host cells or tissues and is therefore tightly regulated by a large family of host plasma (factor $\mathrm{H}, \mathrm{C} 4 \mathrm{bBP}$ ) and membrane-bound (CD35, CD46, CD55, and CD59) proteins called regulators of complement activation (RCA) that are involved in the physiological control of various stages of the complement cascade. Complement inactivation by RCA has been shown to be species specific (homologous restriction) $(19,44)$. A number of enveloped viruses have been shown to incorporate cellassociated RCA proteins into budding particles $(18,38,39,41)$.

Viruses have been used in cancer treatment for over a century (26). The tepid response to oncolytic virotherapy studies performed in the 1960s and 1990s has undergone resurgence in the past decade with new advances in the manipulation of viral genomes, a more thorough understanding of tumor-host interactions and professionally conducted clinical trials (42). Translating laboratory successes into clinical breakthroughs has, however, been frustratingly slow with only a few oncolytic viruses (OVs) undergoing phase III clinical trials (3). Issues of safety and doseassociated toxicity have proved to be an impediment to U.S. Food and Drug Administration approval of OVs for cancer therapy. Effective systemic administration of both inherently oncolytic and engineered (targeted and transgene armed) viruses offer potentially high levels of antitumor potency, but the host immune sys- tem remains a significant obstacle to targeted tumor delivery. Neutralizing antibodies in serum, the complement system or uptake by reticuloendothelial cells reduce the infectivity of OVs, necessitating high doses or repeated injections (35). Toxic side effects include neurological problems, liver toxicity, leucopenia, or low-grade intravascular coagulation, underscoring the need for alternative approaches to OV administration (45).

Newcastle disease virus (NDV) is a nonsegmented negativestrand member of the family Paramyxoviridae whose natural host range is limited to avian species. NDV is inherently tumor selective and oncolytic. The tumor selective property of NDV, coupled with compelling preclinical and phase I/II studies in human subjects, has identified NDV as an attractive virotherapy candidate $(9$, 24, 27).

Strategies to improve the therapeutic index of NDV have been explored in only a few studies $(14,25)$. In addition, the optimal dose, treatment schedule, and routes of administration have not been well defined (27). High mean tolerated doses raise issues of safety $(24,27,42)$. The impact of preexisting immunity to systemic administration with NDV has also not been examined. Here, we address the question of how innate antiviral immunity can limit the efficacy of NDV-based OVs.

NDV is generally propagated in embryonated chicken eggs because of the high virus yield and purified before administration as cancer therapy (9). Experiments in the 1950s and 1970s showed that NDV grown in embryonated chicken eggs could interact with human complement in the absence of detectable antiviral antibody and cause virus inactivation $(11,13,48)$. However, molecu-

\footnotetext{
Received 9 April 2012 Accepted 4 September 2012

Published ahead of print 12 September 2012

Address correspondence to Elankumaran Subbiah, kumarans@vt.edu.

Copyright @ 2012, American Society for Microbiology. All Rights Reserved.

doi:10.1128/JVI.00886-12
} 
lar mechanisms underlying complement activation by NDV have not been precisely elucidated.

In the present study, we examined mechanisms of complement activation by NDV grown in developing chicken embryos. Both classical/lectin and alternative complement pathways mediated the neutralization of NDV. We established that NDV propagated in human cell lines and the World Health Organization-approved primate cell line (Vero) was resistant to complement mediated neutralization through the incorporation of RCA proteins CD46 and CD55. These molecules could enable the virus to evade host innate immune responses, thereby enhancing systemic delivery of the $\mathrm{OV}$ and enabling virus dose reduction.

\section{MATERIALS AND METHODS}

Cells and virus. HeLa human epithelial carcinoma and Vero monkey kidney epithelial cells were grown and maintained in Dulbecco modified Eagle medium supplemented with $10 \%$ heat-inactivated fetal bovine serum, $100 \mu \mathrm{g}$ of penicillin/ml, and $0.1 \mu \mathrm{g}$ of streptomycin (Invitrogen, Carlsbad, CA)/ml. Chinese hamster ovary (CHO) cells, stably transfected with the CYT2 isoform of human CD46 (CHO 5.3), were initially generated by Loveland et al. (28) and acquired from Denis Gerlier, University of Lyon. The cells were maintained in minimal essential medium supplemented, as described above, with $1 \%$ nonessential amino acids and $500 \mu \mathrm{g}$ of Geneticin (Invitrogen)/ml. Control CHO K1 cells were grown in the absence of Geneticin. Full-length infectious clone of NDV strain LaSota was a gift from Siba K. Samal, University of Maryland, College Park, MD. An extra cistron-encoding enhanced green fluorescent protein (eGFP) was inserted between the phosphoprotein $(\mathrm{P})$ and matrix $(\mathrm{M})$ gene sequences of full-length infectious clone, as described elsewhere (7). The recombinant virus (rLaSota eGFP) was propagated in the allantoic fluid of 9-day-old embryonated specific-pathogen-free chicken eggs, concentrated by ultracentrifugation, and purified on a 10 to $26 \%$ Opti-Prep gradient (Axis-Shield PoC, Oslo, Norway) by centrifugation at 27,000 rpm for $2 \mathrm{~h}$ in a Beckman Coulter Optima L-90K ultracentrifuge. HeLa, Vero, $\mathrm{CHO} \mathrm{K} 1$, and $\mathrm{CHO} 5.3$ cell monolayers were infected at multiplicity of infection (MOI) of 0.01 with rLaSota eGFP in the presence of $1 \mu \mathrm{g}$ of TPCK (tolylsulfonyl phenylalanyl chloromethyl ketone)-treated trypsin (Sigma-Aldrich, St. Louis, MO)/ml. Infected cell supernatants were concentrated and purified as described above. rLaSota eGFP derived from allantoic fluid or cell lines were termed NDV/egg, NDV/CHO K1, NDV/ $\mathrm{CHO} 5.3$, NDV/HeLa, and NDV/Vero, respectively. Virus titers were determined by fluorescent plaque assay on Vero cell monolayers as described elsewhere (40).

Virus neutralization. Normal human serum (NHS) and normal chicken serum (NCS) samples were tested for 50\% total hemolytic complement activity $\left(\mathrm{CH}_{50}\right)$ using antibody-sensitized sheep erythrocytes (Complement Technology, Inc., Tyler, TX). Totals of 100 PFU of NDV/ egg, NDV/CHO K1, NDV/CHO 5.3, NDV/HeLa, and NDV/Vero, respectively, were treated with various dilutions of NHS or NCS in phosphatebuffered saline $\left(\mathrm{Ca}^{2+}, \mathrm{Mg}^{2+}\right)$ by incubation at $37^{\circ} \mathrm{C}$ for $1 \mathrm{~h}$ or various time intervals $(5,10,15,20,30,45$, and $60 \mathrm{~min})$. Virus-serum mixtures were then overlaid on Vero cell monolayers, and fluorescent plaques were visualized after $72 \mathrm{~h}$ under a UV light source. The plaques were normalized relative to $100 \mathrm{PFU}$ in untreated virus controls, with neutralization by complement represented as the number of plaques below $100 \mathrm{PFU}$. In a separate experiment, $100 \mathrm{PFU}$ of NDV/egg were incubated with NHS treated with either $3 \mathrm{mM}$ EDTA or $8 \mathrm{mM}$ EGTA plus $2 \mathrm{mM} \mathrm{MgCl}_{2}$ for $1 \mathrm{~h}$ at $37^{\circ} \mathrm{C}$. NHS heat treated at $56^{\circ} \mathrm{C}$ for $1 \mathrm{~h}$ (HT-NHS) and untreated NHS served as controls for complement activation. Virus-serum mixtures were overlaid on Vero cell monolayers and quantified as described above.

In order to test the presence of naturally occurring antibodies to Gal $\alpha 1-3$ Gal sugars on NDV/egg, NHS samples were preincubated for $1 \mathrm{~h}$ at $37^{\circ} \mathrm{C}$ with $10 \mathrm{mg}$ of Gal 1 1-3Gal disaccharides (Toronto Research Chemicals, Inc., Toronto, Canada)/ml as described elsewhere (49). NHS samples were then incubated with $100 \mathrm{PFU}$ of NDV/egg, overlaid on Vero cell monolayers, and quantified as described above.

Depletion and reconstitution of complement components. A total of $100 \mathrm{PFU}$ of NDV/egg were treated with various dilutions of NHS depleted of $\mathrm{C} 2, \mathrm{C} 3$, or $\mathrm{C} 4$ (Complement Technology) by incubation for $1 \mathrm{~h}$ at $37^{\circ} \mathrm{C}$, followed by overlaying on Vero cells and quantification by plaque assay. In a set of reconstitution experiments, various dilutions of NHS depleted of C2, C3, or C4 were reconstituted with physiological concentrations of purified C2 $(25 \mu \mathrm{g} / \mathrm{ml})$, C3 $(1.3 \mathrm{mg} / \mathrm{ml})$, or C4 $(200 \mu \mathrm{g} / \mathrm{ml})$ (Complement Technology) before incubation with $100 \mathrm{PFU}$ of NDV/egg and subsequent quantification by plaque assay.

Transmission electron microscopy. Gradient-purified rLaSota eGFP particles (NDV/egg) were analyzed for C3 deposition by adsorption of $10^{5}$ viral particles on carbon-coated 200-mesh gold grids (Electron Microscopy Sciences, Hatfield, PA) after incubation at room temperature in a humidified chamber for $5 \mathrm{~min}$. Grids were blocked with phosphate-buffered saline (PBS) containing 1\% bovine serum albumin, and adsorbed virus was treated with 1:25 diluted C8-depleted NHS (Complement Technology), followed by mouse anti-human C3/C3b monoclonal antibody (Cell Sciences, Inc., Canton, MA). Bound antibody was detected using goat anti-mouse antibody conjugated with 12-nm colloidal gold (Jackson ImmunoResearch Laboratories, West Grove, PA). Labeled particles were negatively stained with $2 \%$ phosphotungstic acid ( $\mathrm{pH} 6.6$ ) and analyzed under a Tecnai Spirit transmission electron microscope with an AMT Dual 2Vu camera (FEI, Hillsboro, OR).

Purified NDV/HeLa was analyzed for CD46 and CD55 incorporation on virion surfaces by adsorption on grids as described above. After blocking, adsorbed viruses were probed with mouse anti-human CD46 (R\&D Systems, Minneapolis, MN) and mouse anti-human CD55 antibodies, respectively (Millipore, Billerica, MA) and detected with secondary 6-nm colloidal gold-conjugated goat anti-mouse antibody to CD46 and 12-nm colloidal gold-conjugated secondary antibody to CD55 (Jackson ImmunoResearch Laboratories). Labeled particles were negatively stained and visualized as described above.

C3b cofactor assay. Ten thousand PFU of the NDV/egg, NDV/HeLa, and NDV/Vero were incubated for various time intervals at $37^{\circ} \mathrm{C}$ with 3 $\mu \mathrm{g}$ of purified C3b and $100 \mathrm{ng}$ of factor I (Complement Technology) in a total volume of $20 \mu$ l. Reactions were terminated by boiling in SDS-PAGE sample buffer, and the samples were subjected to electrophoresis on $8 \%$ polyacrylamide gels (Bio-Rad, Hercules, CA). Protein bands were visualized by staining with Coomassie brilliant blue R-250. Factor $\mathrm{H}$ served as a positive control for factor I-mediated cleavage of C3b into iC $3 \mathrm{~b}$.

ELISA. A total of $1,000 \mathrm{PFU}$ of NDV/egg were incubated at $37^{\circ} \mathrm{C}$ with a 1:1,000 dilution of NHS in PBS $\left(\mathrm{Ca}^{2+}, \mathrm{Mg}^{2+}\right)$ for various time intervals $(0,15,30$, and $60 \mathrm{~min})$. NHS incubated with only PBS for the same time intervals served as a negative control. Reactions were terminated by incubating the virus-serum mixtures on ice, and the complement activity was quantitated using the human $\mathrm{C} 3 \mathrm{a}, \mathrm{C} 4 \mathrm{a}$, or $\mathrm{C} 5 \mathrm{a}$ enzyme-linked immunosorbent assay (ELISA) kits (BD Biosciences, San Jose, CA) according to the manufacturer's instructions.

Density gradient centrifugation and immunoblotting. A total of $100,000 \mathrm{PFU}$ of NDV/egg were treated with undiluted NHS by incubation at $37^{\circ} \mathrm{C}$ for $1 \mathrm{~h}$. The virus-serum mixture was layered on a 10 to $26 \%$ Opti-Prep gradient, and density gradient centrifugation was carried out at 26,000 rpm (SW41 rotor, $2 \mathrm{~h}, 4^{\circ} \mathrm{C}$ ) in a Beckman Coulter Optima L-90K ultracentrifuge. Fractions $(250 \mu \mathrm{l})$, starting from the bottom of the gradient, were removed and analyzed for the presence of virus and C3 by immunoblotting. Virus-only and serum-only samples were also centrifuged as controls. Fractions collected were probed for virus using NDV polyclonal serum raised in chicken. C3 was detected using a goat antihuman C3 polyclonal antibody (Complement Technology).

CHO K1, CHO 5.3, HeLa, and Vero cells were harvested in radioimmunoprecipitation assay lysis buffer (Millipore) containing halt protease inhibitor cocktail (Pierce Biotechnology, Rockford, IL). Total protein content was quantified with the Micro BCA assay (Pierce Biotechnology). 

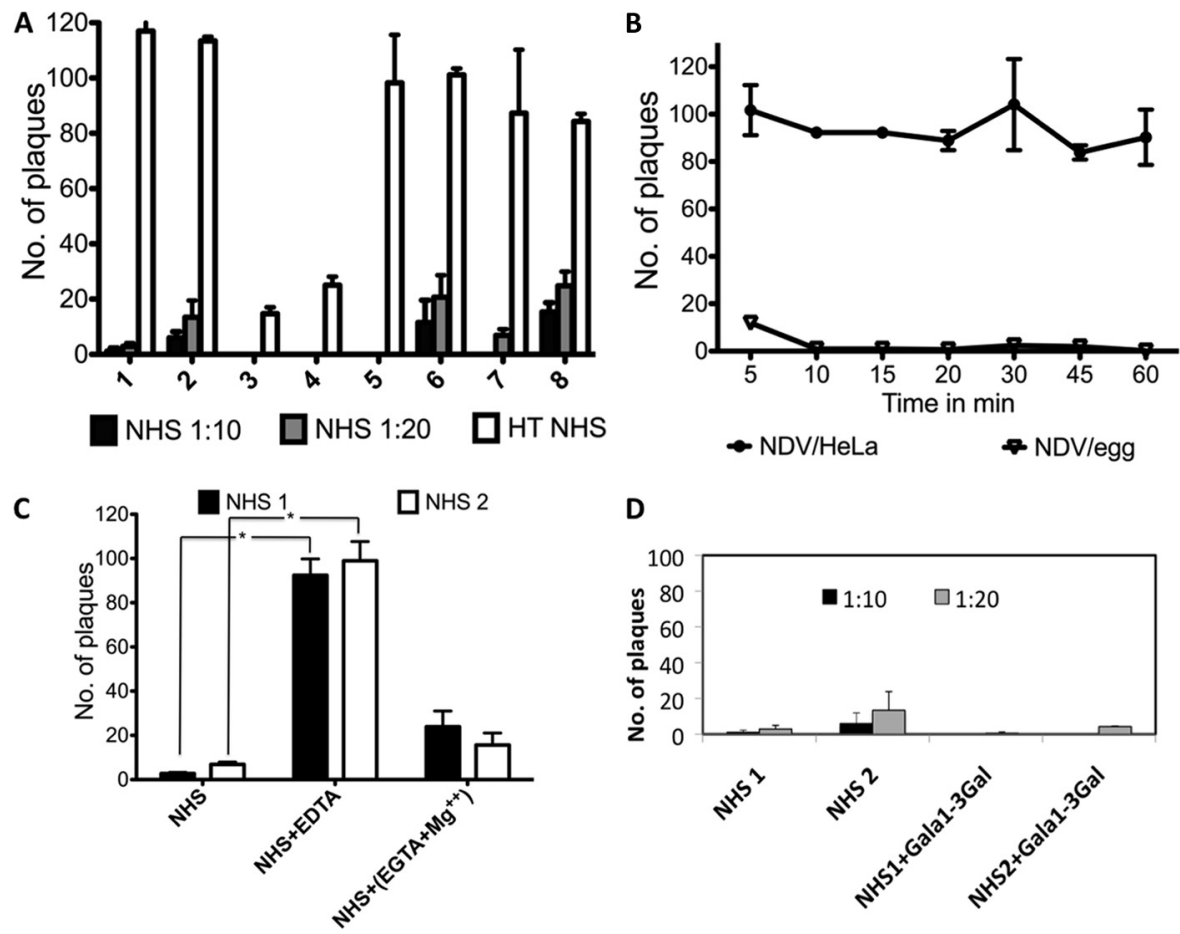

FIG 1 Multiple complement pathways in normal human serum (NHS) neutralize NDV infectivity. (A) Dilutions (1:10 and 1:20) of NHS and heat-treated NHS (HT-NHS) from eight human donors were incubated with $100 \mathrm{PFU}$ of egg-grown NDV (NDV/egg). The remaining infectivity following incubation was determined by a fluorescent plaque assay on Vero cells. (B) Totals of $100 \mathrm{PFU}$ of NDV/egg and NDV/HeLa were incubated with 1:10 diluted NHS for a time course of 5 to $60 \mathrm{~min}$. The remaining virus infectivity was determined by a fluorescent plaque assay on Vero cells. Each point represents the mean + the standard deviation (SD). (C) NHS, EDTA-treated NHS, or EGTA- and $\mathrm{Mg}^{2+}$-treated NHS of donors 1 and 2 from panel A were incubated with 100 PFU of NDV. The remaining virus infectivity was determined by plaque assay. Bars represent the means + the SDs. Significant differences between untreated and EDTA-treated NHS are represented by asterisks $\left({ }^{*}\right)$. EDTA treatment abolished complement-mediated neutralization of NHS $(P<0.05$, paired sample $t$ test). (D) NHS of donors 1 and 2 were preincubated with Gal $\alpha 1-3 \mathrm{Gal}$ disaccharides prior to virus neutralization assay with $100 \mathrm{PFU}$ of NDV. The remaining virus infectivity was determined by a fluorescent plaque assay. NHS-only samples were used as a control in order to compare virus neutralization titers.

A $20-\mu$ g portion of protein was loaded onto a $10 \%$ polyacrylamide gel (Bio-Rad) and transferred to a polyvinylidene difluoride membrane using the iBlot gel transfer system (Invitrogen). Membranes were blocked in 5\% skim milk and probed with a polyclonal antibody that was cross-reactive to human, mouse, and rat CD46 and a monoclonal antibody that was specific for human CD55 (clone H-7; Santa Cruz Biotechnology, Santa Cruz, CA). Antibody to actin and horseradish peroxidase-conjugated species-specific secondary antibodies were also used (Santa Cruz Biotechnology). In addition, 10,000 PFU of purified NDV/CHO K1, NDV/CHO 5.3, NDV/HeLa, and NDV/Vero were probed for viral antigen and RCA molecules CD46 and CD55. Protein bands were visualized on X-ray film (Phenix Research Products, Candler, NC) using the Super Signal West Pico chemiluminescent substrate (Pierce Biotechnology).

\section{RESULTS}

NHS selectively neutralizes NDV grown in embryonated chicken eggs in the absence of virus-specific or natural antibodies. NHS samples from eight individual donors with known complement activity $\left(\mathrm{CH}_{50}\right.$ values of $\left.250 \mathrm{U} / \mathrm{ml}\right)$ were incubated with $100 \mathrm{PFU}$ of purified NDV/egg, and the remaining infectivity was measured by a plaque assay on Vero cells. Both 1:10 and 1:20 dilutions of NHS in PBS reduced the number of plaques by $>80 \%$ (Fig. 1A). This ability to neutralize infectivity was lost upon heat treatment for all but two sera (sera 3 and 4), indicating that the activation of human complement was responsible. Hemagglutination inhibition (HI) analysis of these sera revealed the presence of neutralizing antibodies to NDV in sera 3 and 4 (HI titer $\geq 40$ ), while the remaining serum samples had no neutralizing antibodies to NDV (HI titer $\leq 10$ ) (data not shown). Serum from donors 1, 2, and 5 were used for all further experiments that required NHS.

A time course of neutralization was performed by incubating $100 \mathrm{PFU}$ of NDV/egg or NDV/HeLa with NHS; the remaining virus infectivity was then quantified by plaque assay. NDV/HeLa was completely resistant to neutralization by NHS at all time points tested, whereas the neutralization of NDV/egg by NHS was seen to be rapid, with $90 \%$ neutralization occurring in the first 5 min of virus-serum incubation and complete neutralization occurring by $10 \mathrm{~min}$ (Fig. 1B).

$\mathrm{Ca}^{2+}$ is required for the activation of the classical and mannose-binding lectin (MBL) pathways and EGTA, which chelates $\mathrm{Ca}^{2+}$, inhibits both of these pathways (6). All three pathways require $\mathrm{Mg}^{2+}$ and are inhibited by the $\mathrm{Ca}^{2+}$ and $\mathrm{Mg}^{2+}$ chelating agent, EDTA (50). Pretreatment of 10\% NHS with 3 mM EDTA resulted in the loss of complement-mediated neutralization of NDV (Fig. 1C). Pretreatment of NHS with 8 mM EGTA supplemented with $2 \mathrm{mM} \mathrm{Mg}^{2+}$ ions resulted in a partial loss of NDV neutralization, suggesting the participation of multiple pathways of complement activation in NDV/egg neutralization.

Several viruses, including lymphocytic choriomeningitis virus and some paramyxoviruses (SV5 and mumps), are neutralized by complement in the absence of virus-specific antibodies $(17,30,38$, 
A

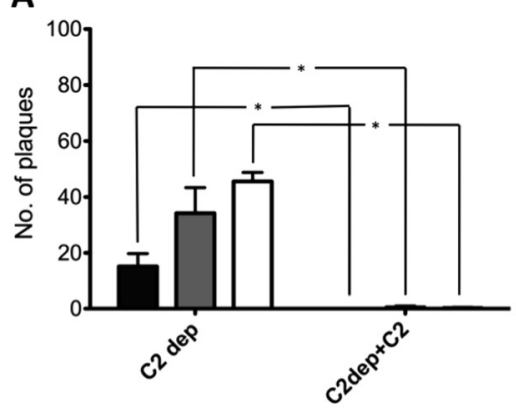

$1: 10$

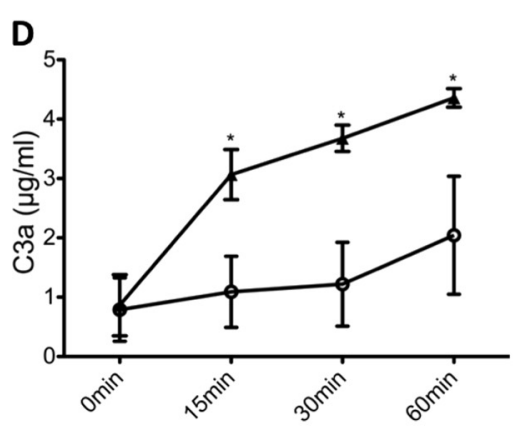

$\bullet$ NHS
B

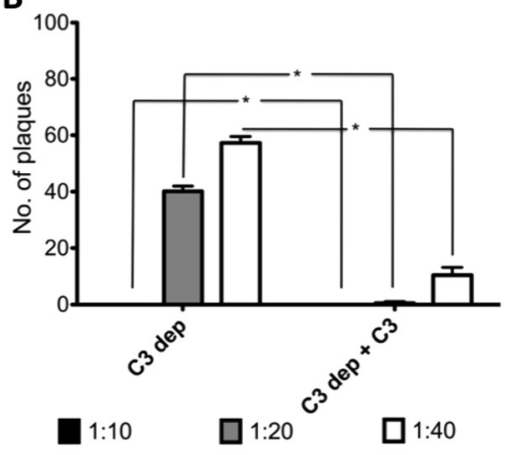

E

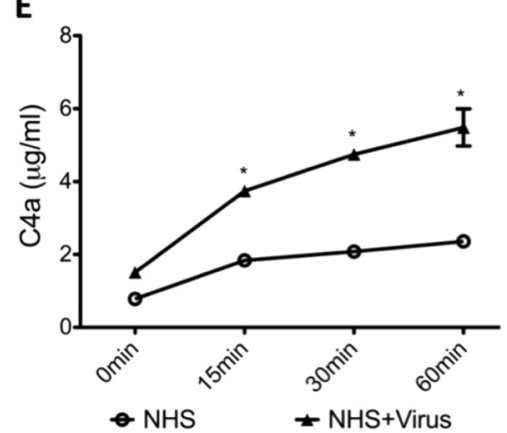

C

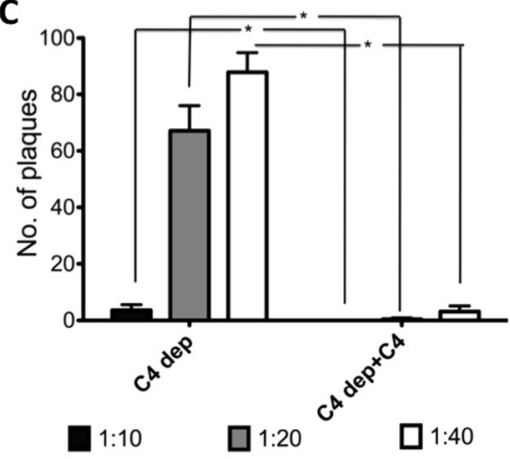

$\mathbf{F}$

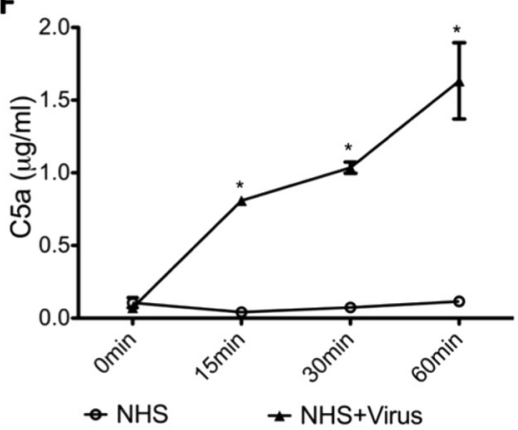

FIG 2 Neutralization of NDV by NHS requires the activity of C2, C4, C3, and C5 components. A total of 100 PFU of NDV/egg was incubated with 1:10, 1:20, and 1:40 dilutions of C2-depleted NHS (A), C4-depleted NHS (B), and C3-depleted NHS (C). C2-, C4-, and C3-depleted sera were also reconstituted with physiological concentrations of purified $\mathrm{C} 2, \mathrm{C} 4$, and $\mathrm{C} 3$ in panels A to $\mathrm{C}$, respectively. The remaining infectivity was determined by a fluorescent plaque assay. Significant differences between depleted, and supplemented sera are indicated by asterisks ${ }^{*}$ ). A significant increase in the number of plaques of C2-, C4-, and C3-depleted NHS was observed compared to supplemented NHS $(P<0.05$, Wilcoxon signed-rank test, Tukey-Kramer HSD). NHS was incubated with NDV/egg for the indicated times in minutes, and the levels of C3a (D), C4a (E), and C5a (F) were quantitated by ELISA. NHS incubated with PBS for the same time points served as a control. Significant differences between NHS incubated with PBS and NHS incubated with NDV/egg are indicated by asterisks $\left({ }^{*}\right)$. Significant increases in the levels of C3a, C4a, and C5a upon incubation with NDV were observed $(P<0.05$, Student $t$ test, Tukey-Kramer HSD). Bars represent the means + the SDs.

39). However, naturally occurring antibodies to Gal $\alpha 1-3$ Gal have also been shown to neutralize virus (49). Pretreatment of NHS with Gal 1 -3Gal disaccharides, as described elsewhere (49), in order to consume anti-Gal natural antibodies in serum had no effect on virus neutralization (Fig. 1D). This suggested that naturally occurring antibodies to Gal $\alpha 1-3 \mathrm{Gal}$ disaccharides did not neutralize NDV/egg and that virus neutralization by NHS in the absence of virus specific antibodies was a result of complement activity.

NDV/egg induces multiple pathways of complement activation in NHS. Complement activation pathways initiated by NDV/ egg were further analyzed using human sera that were deficient in complement factors $\mathrm{C} 2, \mathrm{C} 3$, or C4. Both classical and MBL pathway activation requires the cleavage of $\mathrm{C} 4$ and $\mathrm{C} 2$, which form the C3 convertase, C4b2a. All three pathways converge upon the C3 molecule, which can subsequently lead to $\mathrm{C} 5$ cleavage and generation of the membrane attack complex and viral lysis (2). At 1:20 and 1:40 dilutions, C2-depleted serum reduced the NDV/egg PFU levels by 65 and $55 \%$, respectively. C3-depleted sera reduced NDV/egg PFU by 60 and 43\%, whereas C4-depleted serum reduced the number of NDV/egg plaques by 33 and $14 \%$ at $1: 20$ and 1:40 dilutions, respectively (Fig. 2A to C). C2-, C3-, or C4-deficient sera supplemented with purified C2, C3, or C4, respectively, significantly reduced the number of NDV/egg plaques at 1:10, 1:20, and 1:40 dilutions compared to deficient sera (Fig. 2A to C).
ELISAs for C3a, C4a, and C5a fragments further confirmed that virions were fixing complement (Fig. 2D to F). Incubation of NHS with NDV/egg resulted in the activation and cleavage-mediated release of C3a, C4a, or C5a within $15 \mathrm{~min}$. Control serum incubated with PBS did not release C4a or C5a above background levels at any time point (Fig. 2D to F), except for C3a, in which spontaneous cleavage of $\mathrm{C} 3$ into $\mathrm{C} 3 \mathrm{a}$ and $\mathrm{C} 3 \mathrm{~b}$ fragments is known to occur (34).

Complement activation in NHS is associated with C3 deposition on virions. $\mathrm{C} 3$ deposition on a virus or infected cell surface occurs in an amplification loop that results in neutralization by opsonization, aggregation, phagocytosis, or lysis (37). NDV association with $\mathrm{C} 3$ was analyzed by density gradient centrifugation of NDV/egg incubated with NHS. Immunoblotting for NDV proteins in gradient fractions indicated that untreated NDV/egg virions migrated as a band between fractions 15 to 21, whereas NDV/ egg incubated with NHS revealed a faster-migrating band between fractions 13 and 19, with some sort of virus-serum aggregate at the bottom of the tube that did not include complement $\mathrm{C} 3$ (fractions 1 and 2) (Fig. 3A). Virus antigen was not detected at the top of the gradient, suggesting that the viral membrane had not been released by complement lysis. Immunoblotting of the faster migrating fraction with C3 antibody revealed cosedimentation of C3 fragments in the same fractions that contained viral proteins (fractions 13 to 19, Fig. 3A). C3 fragments were also observed in lower 

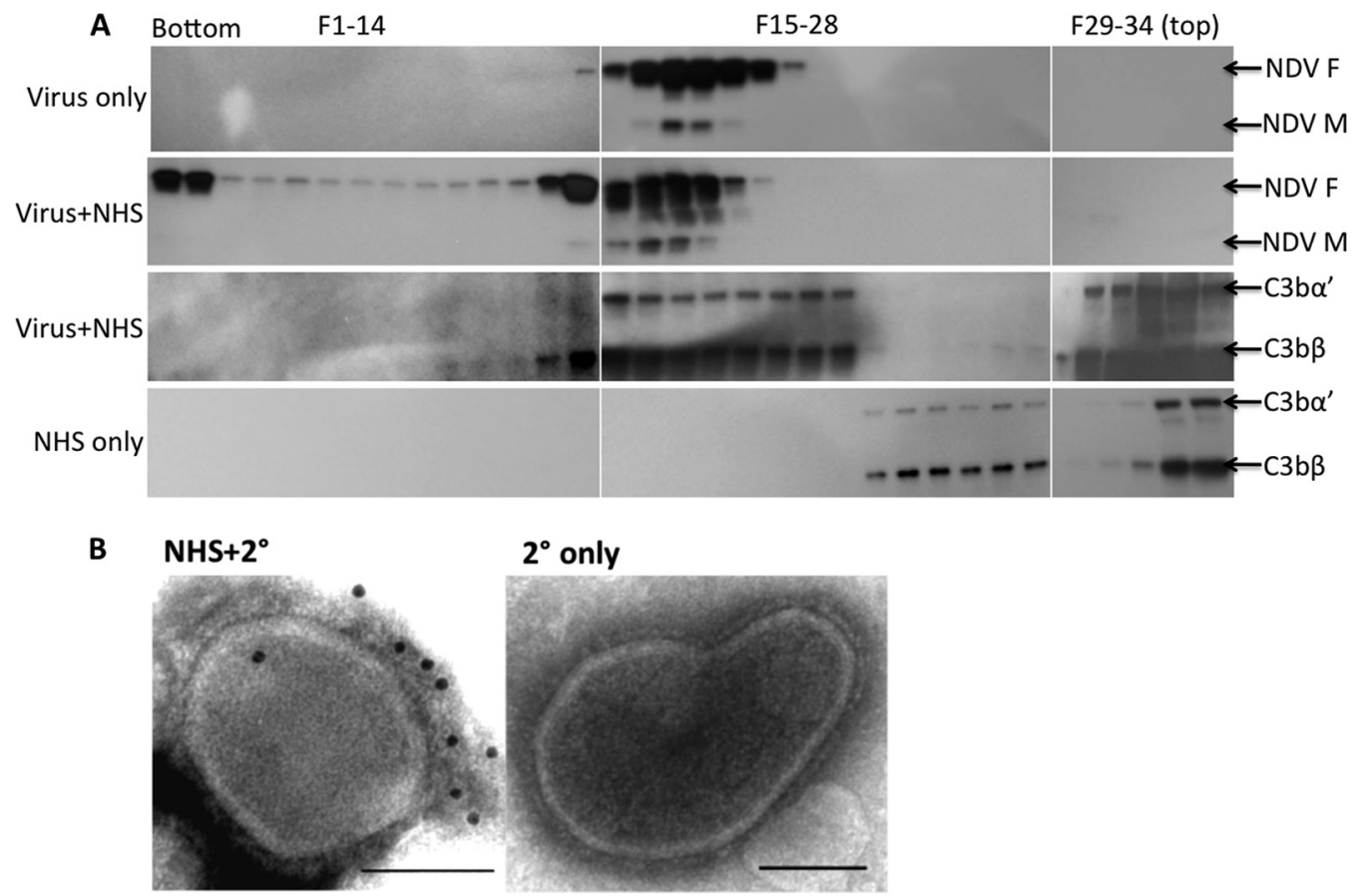

FIG 3 Neutralization of NDV by complement proceeds by C3 deposition. (A) Purified NDV/egg was incubated with NHS and overlaid on a 10 to $26 \%$ Opti-Prep gradient, and fractions were collected from the bottom following density gradient centrifugation. Virus-only and virus-NHS (Virus + NHS) fractions were immunoblotted with NDV polyclonal sera: bands specific for the NDV fusion (F) and matrix (M) protein were detected. Virus-NHS fractions were also immunoblotted for the C3 protein. NHS-only fractions were also probed for C3. The positions of C3b $\alpha^{\prime}$ and C3b $\beta$ are indicated. (B) Purified NDV/egg particles were treated with 1:25-diluted C8-depleted serum. C3 deposition was detected with a primary C3 antibody and secondary 12-nm colloidal gold-conjugated species-specific antibody. Transmission electron microscopic analysis show colloidal gold binding to NHS-treated particles NHS- $2^{\circ}()$, whereas no binding is visible on the control treated with only secondary ( $2^{\circ}$ only) antibody. Scale bars, $0.1 \mu \mathrm{m}$.

density fractions that did not include obvious viral proteins (fractions 20 to 22, Fig. 3A), which could be released membranes of an intermediate density with $\mathrm{C} 3$ deposited on them. C3b $\alpha^{\prime}$ (110$\mathrm{kDa})$ and $\mathrm{C} 3 \mathrm{~b} \beta(75-\mathrm{kDa})$ bands and the remaining NHS proteins were observed at the top of the gradient. Gradient centrifugation of only NHS, in the absence of virus, showed C3 fragments only at the top of the gradient (Fig. 3A). In addition, C3 deposition on the surfaces of NDV/egg particles was observed by immunogold electron microscopy labeling, even after a short incubation period of 5 min (Fig. 3B). Virus incubated with gold-conjugated secondary antibody alone was not labeled.

NDV incorporates membrane RCA into viral envelopes. NDV grown in HeLa (NDV/HeLa), a human cell line, displayed resistance to virus neutralization by NHS (Fig. 1B). To determine whether this resistance was associated with the incorporation of membrane-bound RCA proteins into viral envelopes, immunoblotting and immunogold electron microscopy experiments were carried out. NDV was grown in HeLa and Vero cells and, in addition, $\mathrm{CHO} \mathrm{K} 1$ cells that either displayed no intrinsic regulatory activity on their cell surfaces for human $\mathrm{C} 3 \mathrm{~b}$ or $\mathrm{C} 4 \mathrm{~b}$ and recombinant $\mathrm{CHO} \mathrm{K} 1$ cells that expressed human $\mathrm{CD} 46$ (CHO 5.3). CHO 5.3 cells expressed a lower-molecular-weight isoform of CD46 compared to HeLa and Vero cells (Fig. 4A). NDV/Vero and NDV/CHO 5.3 incorporated only the CD46 molecule onto virions, whereas NDV/HeLa incorporated both CD46 and CD55 (Fig. $4 \mathrm{~A})$. Immunogold electron microscopy on purified NDV/HeLa particles confirmed labeling for both CD46 and CD55 on the viral envelope (Fig. 4B).
No band corresponding to CD55 was detected in NDV/CHO $\mathrm{K} 1$, although a faint band corresponding to the lower-molecularweight isoform of CD46 was observed. The absence of RCA detection in $\mathrm{CHO} \mathrm{K} 1$ could be because the monoclonal antibody to human CD55 could not detect the hamster homolog of CD55, whereas the polyclonal antibody to human, mouse, and rat CD46 probably reacted in a cross-species manner with hamster CD46.

CD46 and CD55 molecules on virions exhibit homologous restriction. Complement evasion by incorporation of membrane bound RCAs onto virion surfaces was quantified by plaque assay. Purified NDV/HeLa, NDV/Vero, NDV/CHO K1, NDV/CHO 5.3, or NDV/egg (100 PFU) were incubated with various dilutions of NHS or NCS, and the remaining infectivity was determined by plaque assay. As observed earlier, NDV/egg was neutralized by NHS, whereas NDV/HeLa, NDV/Vero, and NDV/CHO 5.3 were resistant to complement-mediated neutralization to various degrees (Fig. 5A). Resistance to complement was complete with NDV/HeLa virions. Significant resistance to complement-mediated neutralization was also observed at 1:20 and 1:40 dilutions of NHS with NDV/Vero and NDV/CHO 5.3, whereas ca. 90\% infectivity was retained at 1:40 dilution. Resistance to NHS complement neutralization by NDV/HeLa and NDV/CHO 5.3 was not observed on incubation with NCS, whereas NHS complement and NCS complement neutralized NDV/CHO K1 equally, probably because of homologous restriction of hamster RCA (Fig. 5B). In contrast, NDV/egg was resistant to complement-mediated neutralization by NCS. These results confirm the species specificity of 
A

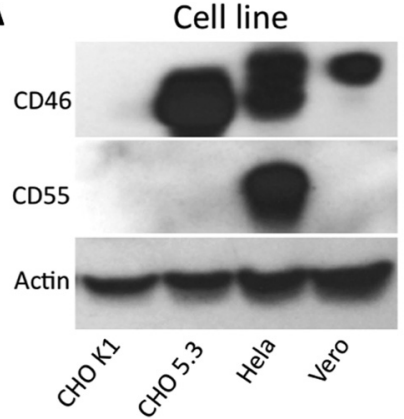

B

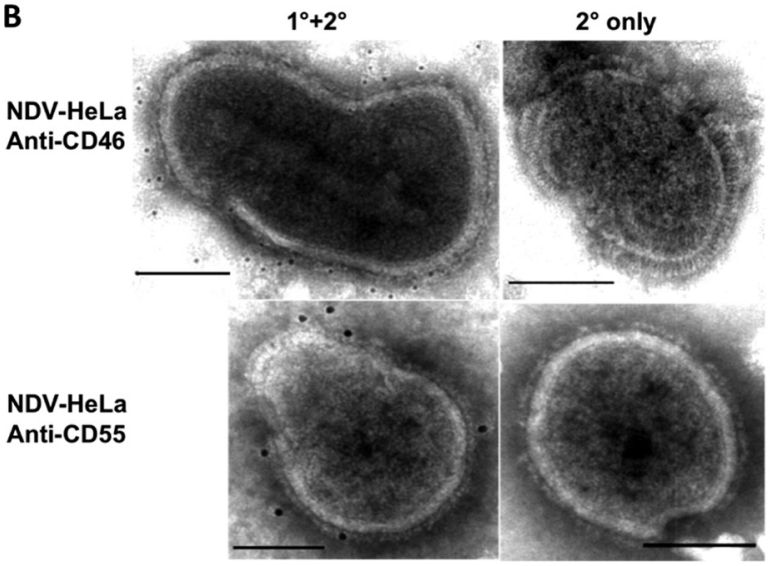

FIG 4 Incorporation of RCA molecules by NDV grown in mammalian cells. (A) Lysates of CHO K1, CHO 5.3, HeLa, and Vero cells and the purified viruses grown in them were immunoblotted with antibodies to CD46 and CD55. Protein bands of actin and the NDV matrix (2) protein served as loading controls for cell and virus samples, respectively. (B) Purified NDV/HeLa was probed with anti-CD46 or CD55 antibody and detected with secondary 6- and $12-\mathrm{nm}$ colloidal gold-conjugated goat anti-mouse antibody, respectively $\left(1^{\circ}+2^{\circ}\right)$. Virions treated with secondary antibody only served as control $\left(2^{\circ}\right.$ only). Scale bars, $0.1 \mu \mathrm{m}$.

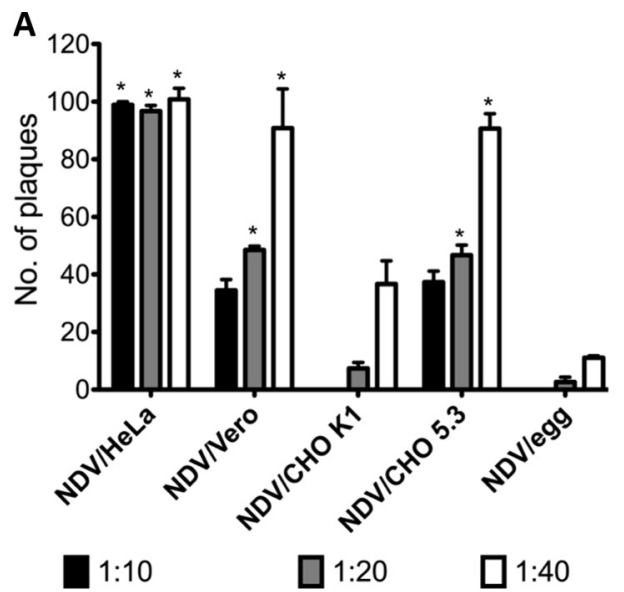

RCA molecules and, in addition, strongly indicate the incorporation of chicken-specific RCA components by NDV/egg.

NDV grown in mammalian cells inactivates human $\mathrm{C} 3 \mathrm{~b}$. C3b deposition on activating surfaces can initiate immune responses and is therefore tightly controlled (37). Factor I, a serine protease, is a regulatory component of both alternative and the classical complement pathways. C3b is rapidly split in two positions by factor I and another cofactor, such as the fluid-phase serum glycoprotein cofactor $\mathrm{H}$ and the membrane-bound cofactors CD35 (CR1) and CD46 (MCP), to form iC3b (67- and 43-kDa fragments) and C3f and finally $\mathrm{C} 3 \mathrm{c}$ and $\mathrm{C} 3 \mathrm{dg}$, thus rendering $\mathrm{C} 3 \mathrm{~b}$ unable to participate in downstream events of complement activation $(1,12)$.

To assess the C3b cofactor activity of purified NDV from different cell sources, we incubated the virus with $\mathrm{C} 3 \mathrm{~b}$ and factor I for various times at $37^{\circ} \mathrm{C}$. As a positive control for $\mathrm{C} 3 \mathrm{~b}$ cofactor activity, C3b was incubated with factor I and the physiologic regulator of complement, factor $\mathrm{H}$. Incubation of purified C3b with factor I and factor $\mathrm{H}$ for $1 \mathrm{~h}$ resulted in the cleavage of $\mathrm{C} 3 \mathrm{~b} \alpha$ into 67- and $43-\mathrm{kDa}$ iC $3 \mathrm{~b}$ fragments, while the $75-\mathrm{kDa} \mathrm{C} 3 \mathrm{~b} \beta$ fragment remained intact (Fig. 6). A time course for iC3b appearance showed that both NDV/HeLa and NDV/Vero inactivated C3b in the presence of factor I in as little as $1 \mathrm{~h}$, whereas purified virions without factor I did not inactivate C3b (Fig. 6A and B). In contrast, NDV/egg did not inactivate C3b in the presence of factor I, and iC $3 \mathrm{~b}$ was observed only on prolonged incubation for $12 \mathrm{~h}$ (Fig. 6C). These results show that NDV proteins by themselves are incapable of inactivating C3b into iC3b. Rather, the incorporation of membrane-bound complement regulator proteins from producer cells into the viral envelope might account for the selective inactivation of human C3b by NDV/HeLa and NDV/Vero.

\section{DISCUSSION}

Recent advances in the field of oncolytic viral therapy has attracted the interest of biopharmaceutical companies $(8,20)$. The development of natural viruses or novel recombinants with high therapeutic index and specificity has been encouraging. Oncolytic po-

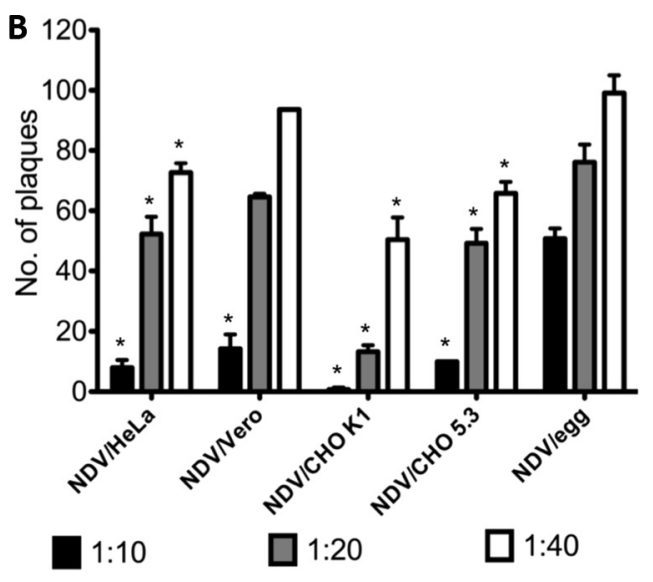

FIG 5 NDV resists complement-mediated neutralization in a species-specific manner. Purified NDV/HeLa, NDV/Vero, NDV/CHO K1, NDV/CHO 5.3, and NDV/egg were incubated for $1 \mathrm{~h}$ at $37^{\circ} \mathrm{C}$ with 1:10, 1:20, and 1:40 dilutions of normal human serum (NHS) (A) and chicken serum (NCS) (B). The remaining virus infectivity was quantified by a fluorescent plaque assay on Vero cells. Bars represent means + the SDs. Significant differences between NDV/egg incubated with NHS or NCS and NDV/HeLa, NDV/Vero, NDV/CHO K1, or NDV/CHO 5.3 incubated with NHS or NCS are indicated by asterisks $\left(^{*}\right)$. Significance was determined by one-way analysis of variance and Tukey-Kramer HSD using NDV/egg as a control according to Dunnett's method. 
A
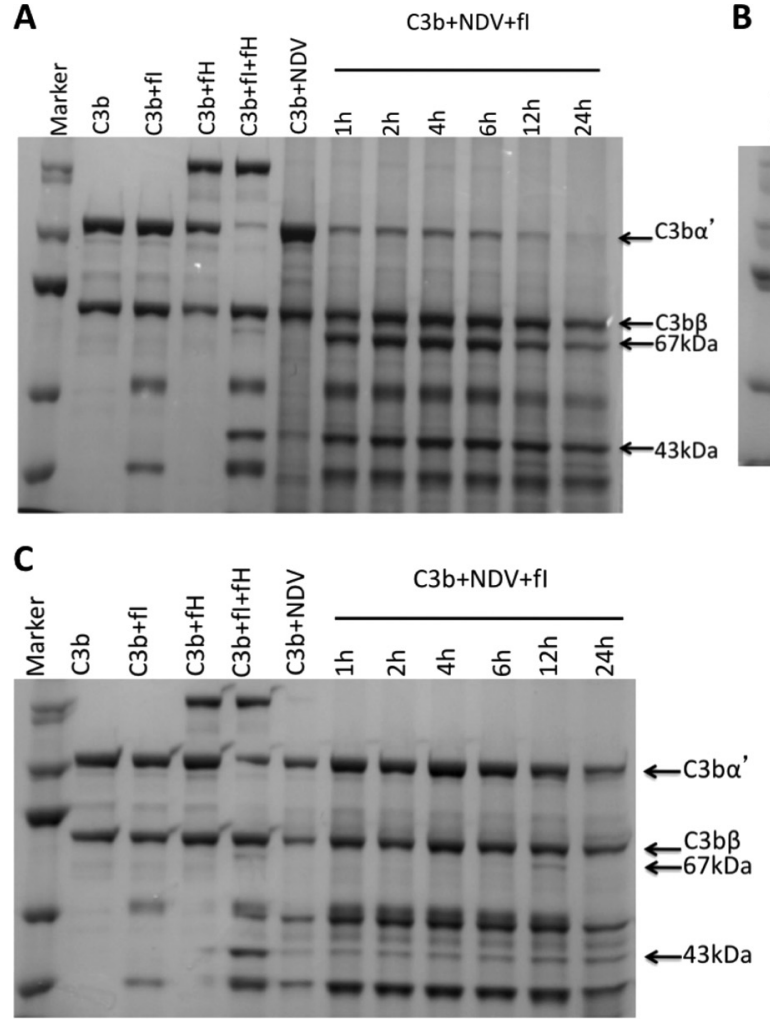

B

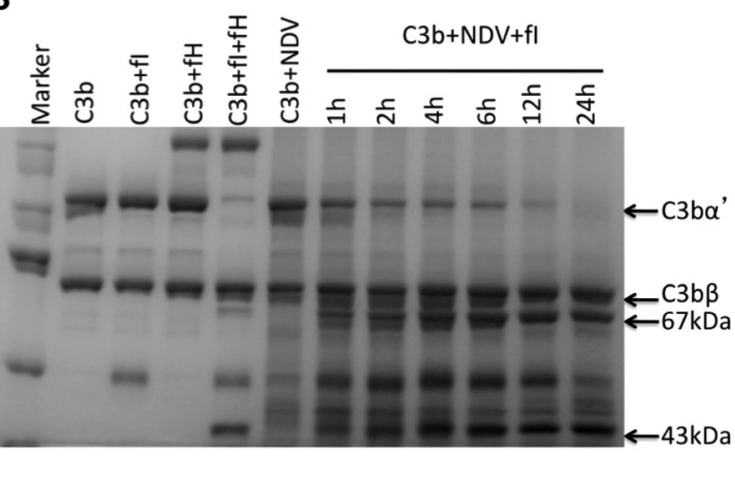

FIG 6 NDV grown in mammalian cells acquire human C3b cofactor activity. Purified NDV/HeLa (A), NDV/Vero (B), and NDV/egg (C) were incubated for 1 to $24 \mathrm{~h}$ with purified human complement component $\mathrm{C} 3 \mathrm{~b}$ and factor I. Lane 1, molecular mass marker; lanes 2 to 5 , controls indicating that C3b cleavage to inactivated $\mathrm{C} 3 \mathrm{~b}(\mathrm{iC} 3 \mathrm{~b})$ requires both factor I and cofactor $\mathrm{H}$; lane 6 , NDV incubated with only C3b; lanes 7 to 12 , incubation of purified NDV with C3b and factor I for various time points. The positions of $\mathrm{C} 3 \mathrm{~b} \alpha$ and $\mathrm{C} 3 \mathrm{~b} \beta$ are indicated. Cleavage of $\mathrm{C} 3 \mathrm{~b} \beta$ into the iC $3 \mathrm{~b}$ fragments of 67 and $43 \mathrm{kDa}$ is observed for $\mathrm{NDV} / \mathrm{HeLa}$ and NDV/Vero.

tential has been somewhat tempered by host antiviral and innate immune responses (23). Although the host immune response may in many ways augment tumor therapy (36), evasion of neutralizing antibodies or complement is the key to achieving efficacy following systemic delivery $(15,46)$. Most oncolytic viruses, including paramyxoviruses, have been shown to activate complement-mediated host defenses, although little is known of this response in the context of oncolytic therapy of tumors (10, $17,47)$. Evasive strategies such as xenogeneic or syngeneic cell delivery of oncolytic viruses into target tissues have been explored for herpesvirus, adenovirus, and vesicular stomatitis virus, among others $(5,21,35)$. However, there have been no attempts to increase the vector potential of oncolytic viruses following systemic delivery.

This study describes the mechanism of NDV neutralization by complement activity in vitro, while investigating strategies that would improve the systemic delivery of NDV both as an oncolytic virus and as a vaccine vector $(23,32)$. We observed that NDV propagated in embryonated chicken eggs activated the complement response in human sera that was dependent on C2, C3, C4, and C5 components. EGTA-treated sera did not completely neutralize NDV, indicating that the classical/lectin pathway works in concert with the alternative pathway of complement activation. It is possible that the initiation of complement response by the classical/lectin pathway is amplified through the alternate pathway. On the other hand, the alternate pathway could mask the effects of the classical/lectin pathway. The activation of more than one complement pathway has been observed earlier in mice infected with West Nile virus (30). Complement activation was observed to be independent of NDV specific antibodies. We also did not find evidence that natural antibodies to Gal $\alpha 1-3 \mathrm{Gal}$ disaccharides were involved in virus neutralization, since blocking of natural antibodies in NHS by incubation with Gal $\alpha 1-3 \mathrm{Gal}$ disaccharides did not affect virus neutralization. Early reports also found that the expression of Gal $\alpha 1-3 \mathrm{Gal}$ epitopes did not alter the sensitivity of NDV to complement-mediated inactivation $(48,49)$.

$\mathrm{C} 3$ is common to all pathways of complement. Coating of $\mathrm{C} 3 \mathrm{~b}$ molecules on viral surfaces can result in activation of complement and lysis of the virion membrane. Alternatively, viral neutralization can occur by opsonization or interference with the viral attachment protein so that it is unable to bind its cellular receptor (31). We observed the association of C3 with NDV virions by cosedimentation of NHS with virus and by immunoelectron microscopy. Downstream activation by C3 cleavage was observed through an increase in C5a levels. C5 activation can lead to viral lysis through assembly of the membrane attack complex on the virion envelope or impair virus infectivity by steric hindrance between virus and cell surface receptors (10). Although we did not obtain any evidence of viral lysis by sedimentation analysis, further studies on downstream effector components are required before this can be confirmed. Identification of the surface molecules on NDV that initiate the complement cascade and how activated 
C5 mediates virus neutralization also remain to be determined. C5 activation requires $\mathrm{C} 1$ (or the lectin pathway), C4, C2, and C3, suggesting that any or all of these proteins may contribute to the neutralization of NDV. Which of the viral components of NDV initiate the complement cascade and how activated C5 mediates virus neutralization remains to be determined. It is also possible that activated C5 interacts with one or both of the surface glycoproteins to prevent virus attachment or fusion.

In vitro analysis with purified complement components showed that NDV virions grown in HeLa and Vero cells had C3b cofactor activity, resulting in specific factor I-mediated cleavage of $\mathrm{C} 3 \mathrm{~b}$ into inactive $\mathrm{iC} 3 \mathrm{~b}$. In contrast, egg-grown NDV had negligible $\mathrm{C} 3 \mathrm{~b}$ cofactor activity. This suggested that the virus producer cell contributed to the $\mathrm{C} 3 \mathrm{~b}$ cofactor activity of NDV. This conclusion differs from viral regulatory proteins reported for other viruses such as the VCP or B5R protein of vaccinia virus, glycoprotein C of herpesvirus, or NS1 of West Nile virus $(4,22,44)$. Further observations that NDV can be either susceptible or resistant to complement activation depending on the host producer cell suggest that NDV may, in the course of infection, passively incorporate host RCAs as it buds through the cell membrane.

NDV was found to incorporate complement regulatory proteins glycosylphosphatidylinositol CD55/DAF and CD46. The incorporation of these RCAs at levels that could provide virus protection against complement-mediated neutralization was observed by in vitro neutralization studies. Importantly, transfection of complement activating $\mathrm{CHO} \mathrm{K} 1$ cells with human CD46 was shown to present significantly more resistance to complement neutralization than control $\mathrm{CHO} \mathrm{K} 1$-grown virus. Control $\mathrm{CHO}$ $\mathrm{K} 1$ cells probably express hamster RCAs that were not subject to homologous restriction. After a time course of neutralization with NHS, NDV/HeLa expressing both CD46 and CD55 showed slower kinetics and a clear resistance to neutralization compared to egg-grown NDV.

RCAs act at the $\mathrm{C} 3$ or $\mathrm{C} 4$ level by either accelerating the decay of the bimolecular C3 convertases or as cofactors for factor I, thereby interfering with the deposition of complement on virions (1). A number of enveloped viruses, including the paramyxovirus SV5 (PIV5) and mumps virus, have been shown to incorporate cell-associated RCA proteins into budding particles $(18,29,38)$. In addition, retrovirus and baculovirus as gene delivery vehicles have shown enhanced complement evasion upon incorporation of CD46, CD55, and CD59 from producer cells, representing a step in the development of novel viral vectors for improved gene delivery or oncolysis $(19,38,39,43)$.

RCAs are thought to function in a species-specific manner in order to protect host cells from unregulated complement activation cascades. A comparison of virus-producer cell combinations revealed that HeLa cell-grown virus, which incorporated both CD46 and CD55, presented the most resistance to complement neutralization. CHO 5.3 and Vero cell-grown viruses incorporated the CD46 molecule, and complement resistance by these virions, while significant, was not as effective as that of HeLa cellgrown viruses. Virus derived from $\mathrm{CHO}$ K1 cells was susceptible to complement neutralization by NHS, as was egg-grown NDV. Normal, nonimmune chicken serum was unable to neutralize NDV grown in embryonated chicken eggs, while effectively neutralizing virions derived from HeLa, Vero, and CHO 5.3 cells. Reports have identified RCA in chickens with functions corresponding to C4bp, CD55, or CD46 $(16,33)$, but these RCA medi- ated protection of egg-grown NDV from chicken serum complement and not from human serum. These results confirm the species-specific restriction of RCA molecules. Collectively, these results demonstrate that NDV incorporates host RCAs into progeny virions during virus budding. These regulators are species specific, and a further understanding of their relative contribution to viral evasion of complement can aid in the production of oncolytic viruses that enhance systemic delivery in humans. We found only a 10 -fold difference in virus yield between NDV grown in chicken embryos and NDV grown in HeLa/Vero cells, suggesting that nonavian cells could be successfully used for NDV production. Our results highlight the need to develop OVs that are resistant to immediate complement-mediated neutralization.

\section{ACKNOWLEDGMENTS}

We thank Siba K. Samal for kindly providing the full-length infectious clone of NDV strain LaSota, Stephen R. Werre for help with statistical analysis, and Jagadeeswaran Deventhiran for help with experiments. We thank Denis Gerlier (University of Lyon) for providing the $\mathrm{CHO}$ cell lines.

We declare that we have no competing financial interests.

This study was supported in part by NIH grants AI070528 to E.S. and AI083253 to G.D.P.

\section{REFERENCES}

1. Barilla-LaBarca ML, Liszewski MK, Lambris JD, Hourcade D, Atkinson JP. 2002. Role of membrane cofactor protein (CD46) in regulation of $\mathrm{C} 4 \mathrm{~b}$ and C3b deposited on cells. J. Immunol. 168:6298-6304.

2. Blue CE, Spiller ON, Blackbourn DJ. 2004. The relevance of complement to virus biology. Virology 319:176-184.

3. Breitbach CJ, Reid T, Burke J, Bell JC, Kirn DH. 2010. Navigating the clinical development landscape for oncolytic viruses and other cancer therapeutics: no shortcuts on the road to approval. Cytokine Growth Factor Rev. 21:85-89.

4. Chung KM, et al. 2006. West Nile virus nonstructural protein NS1 inhibits complement activation by binding the regulatory protein factor $\mathrm{H}$. Proc. Natl. Acad. Sci. U. S. A. 103:19111-19116.

5. Coukos G, et al. 1999. Use of carrier cells to deliver a replication-selective herpes simplex virus-1 mutant for the intraperitoneal therapy of epithelial ovarian cancer. Clin. Cancer Res. 5:1523-1537.

6. Des Prez RM, Bryan CS, Hawiger J, Colley DG. 1975. Function of the classical and alternative pathways of human complement in serum treated with ethyleneglycol tetraacetic acid and $\mathrm{MgCl}_{2}$-ethylene glycol tetraacetic acid. Infect. Immunol. 11:1235-1243.

7. Elankumaran S, Rockemann D, Samal SK. 2006. Newcastle disease virus exerts oncolysis by both intrinsic and extrinsic caspase-dependent pathways of cell death. J. Virol. 80:7522-7534.

8. Evans J. 2011. Recent deal highlights hopes for cancer-killing viruses. Nat. Med. 17:268-269.

9. Freeman AI, et al. 2006. Phase I/II trial of intravenous NDV-HUJ oncolytic virus in recurrent glioblastoma multiforme. Mol. Ther. 13:221-228.

10. Friedman HM, Wang L, Pangburn MK, Lambris JD, Lubinski J. 2000. Novel mechanism of antibody-independent complement neutralization of herpes simplex virus type 1. J. Immunol. 165:4528-4536.

11. Ginsberg HS, Pillemer L, Wedgewood RJ. 1956. The properdin system and immunity. VI. The inactivation of Newcastle disease virus by the properdin system. J. Exp. Med. 104:707-725.

12. Gros P, Milder FJ, Janssen BJ. 2008. Complement driven by conformational changes. Nat. Rev. Immunol. 8:48-58.

13. Hirsch RL. 1982. The complement system: its importance in the host response to viral infection. Microbiol. Rev. 46:71-85.

14. Hotte SJ, et al. 2007. An optimized clinical regimen for the oncolytic virus PV701. Clin. Cancer Res. 13:977-985.

15. Ikeda K, et al. 2000. Complement depletion facilitates the infection of multiple brain tumors by an intravascular, replication-conditional herpes simplex virus mutant. J. Virol. 74:4765-4775.

16. Inoue $\mathrm{N}$, et al. 2001. A novel chicken membrane-associated complement regulatory protein: molecular cloning and functional characterization. J. Immunol. 166:424-431. 
17. Johnson JB, Capraro GA, Parks GD. 2008. Differential mechanisms of complement-mediated neutralization of the closely related paramyxoviruses simian virus 5 and mumps virus. Virology 376:112-123.

18. Johnson JB, Grant K, Parks GD. 2009. The paramyxoviruses simian virus 5 and mumps virus recruit host cell CD46 to evade complement-mediated neutralization. J. Virol. 83:7602-7611.

19. Kaname Y, et al. 2010. Acquisition of complement resistance through incorporation of CD55/decay-accelerating factor into viral particles bearing baculovirus GP64. J. Virol. 84:3210-3219.

20. Kirn DH. 2011. Redemption for the field of oncolytic virotherapy. Mol. Ther. 19:627-628.

21. Komarova S, Kawakami Y, Stoff-Khalili MA, Curiel DT, Pereboeva L. 2006. Mesenchymal progenitor cells as cellular vehicles for delivery of oncolytic adenoviruses. Mol. Cancer Ther. 5:755-766.

22. Kotwal GJ, Isaacs SN, McKenzie R, Frank MM, Moss B. 1990. Inhibition of the complement cascade by the major secretory protein of vaccinia virus. Science 250:827-830.

23. Kumar S, Nayak B, Collins PL, Samal SK. 2011. Evaluation of the Newcastle disease virus $\mathrm{F}$ and $\mathrm{HN}$ proteins in protective immunity by using a recombinant avian paramyxovirus type 3 vector in chickens. J. Virol. 85:6521-6534.

24. Lam HY, et al. 2011. Safety and clinical usage of Newcastle disease virus in cancer therapy. J. Biomed. Biotechnol. 2011:718710.

25. Laurie SA, et al. 2006. A phase 1 clinical study of intravenous administration of PV701, an oncolytic virus, using two-step desensitization. Clin. Cancer Res. 12:2555-2562.

26. Liu TC, Galanis E, Kirn D. 2007. Clinical trial results with oncolytic virotherapy: a century of promise, a decade of progress. Nat. Clin. Pract. Oncol. 4:101-117.

27. Lorence RM, et al. 2007. Phase 1 clinical experience using intravenous administration of PV701, an oncolytic Newcastle disease virus. Curr. Cancer Drug Targets. 7:157-167.

28. Loveland BE, Johnstone RW, Russell SM, Thorley BR, McKenzie IFC. 1993. Different membrane cofactor protein (CD46) isoforms protect transfected cells against antibody and complement mediated lysis. Transpl. Immunol. 1:101-108.

29. Magre S, et al. 2004. Reduced sensitivity to human serum inactivation of enveloped viruses produced by pig cells transgenic for human CD55 or deficient for the galactosyl- $\alpha$ (1-3) galactosyl epitope. J. Virol. 78:58125819.

30. Melhop E, Diamond MS. 2006. Protective immune responses against West Nile virus are primed by distinct complement activation pathways. J. Exp. Med. 203:1371-1381.

31. Monteflori DC. 1997. Role of complement and Fc receptors in the pathogenesis of HIV-1 infection. Springer Semin. Immunopathol. 18:371-390.

32. Nakaya T, et al. 2001. Recombinant Newcastle disease virus as a vaccine vector. J. Virol. 75:11868-11873.

33. Oshiumi H, et al. 2005. Regulator of complement activation (RCA) locus in chicken: identification of chicken RCA gene cluster and functional RCA proteins. J. Immunol. 175:1724-1734.

34. Pangburn MK, Müller-Eberhard HJ. 1980. Relation of putative thioester bond in $\mathrm{C} 3$ to activation of the alternative pathway and the binding of $\mathrm{C} 3 \mathrm{~b}$ to biological targets of complement. J. Exp. Med. 152:1102-1114.

35. Power AT, et al. 2006. Carrier cell-based delivery of an oncolytic virus circumvents antiviral immunity. Mol. Ther. 15:123-130.

36. Prestwich RJ, et al. 2009. The case of oncolytic viruses versus the immune system: waiting on the judgment of Solomon. Hum. Gene Ther. 20:11191132.

37. Sahu A, Isaacs SN, Soulika AM, Lambris JD. 1998. Interaction of vaccinia virus complement control protein with human complement proteins: factor I-mediated degradation of $\mathrm{C} 3 \mathrm{~b}$ to $\mathrm{iC}_{3} \mathrm{~b}_{1}$ inactivates the alternative complement pathway. J. Immunol. 160:5596-5604.

38. Saifuddin M, et al. 1997. Human immunodeficiency virus type 1 incorporates both glycosyl phosphatidylinositol-anchored CD55 and CD59 and integral membrane CD46 at levels that protect from complementmediated destruction. J. Gen. Virol. 78:1907-1911.

39. Saifuddin M, et al. 1995. Role of virion-associated glycosylphosphatidylinositol-linked proteins CD55 and CD59 in complement resistance of cell line-derived and primary isolates of HIV-1. J. Exp. Med. 182:501-509.

40. Sen Sharma K, et al. 2010. Efficient fluorescence-based imaging methods for quantifying infectivity of Newcastle disease virus. J. Virol. Methods $163: 390-397$.

41. Spear GT, et al. 1995. Host cell-derived complement control proteins CD55 and CD59 are incorporated into the virions of two unrelated enveloped viruses: human T cell leukemia/lymphoma virus type I (HTLV-I) and human cytomegalovirus (HCMV). J. Immunol. 155:4376-4381.

42. Stanford MM, Bell JC, Vaha-Koskela MJ. 2010. Novel oncolytic viruses: riding high on the next wave? Cytokine Growth Factor Rev. 21:177-183.

43. Takeuchi Y, et al. 1994. Type C retrovirus inactivation by human complement is determined by both the viral genome and the producer cell. J. Virol. 68:8001-8007.

44. Vanderplasschen A, Mathew E, Hollinshead M, Sim RB, Smith GL 1998. Extracellular enveloped vaccinia virus is resistant to complement because of incorporation of host complement control proteins into its envelope. Proc. Natl. Acad. Sci. U. S. A. 95:7544-7549.

45. Vile R, Ando D, Kirn D. 2002. The oncolytic virotherapy treatment platform for cancer: unique biological and biosafety points to consider. Cancer Gene Ther. 9:1062-1067.

46. Vile R, Melcher A. 2011. An intravenous stimulus package for oncolytic therapy. Mol. Ther. 19:1930-1932.

47. Wakimoto H, et al. 2002. The complement response against an oncolytic virus is species-specific in its activation pathways. Mol. Ther. 5:275-282.

48. Welsh RM. 1977. Host cell modification of lymphocytic choriomeningitis virus and Newcastle disease virus altering viral inactivation by human complement. J. Immunol. 118:348-354

49. Welsh RM, O'Donnell CL, Reed DJ, Rother RP. 1998. Evaluation of the Gal $\alpha 1-3 \mathrm{Gal}$ epitope as a host modification factor eliciting natural humoral immunity to enveloped viruses. J. Virol. 72:4650-4656.

50. Zapf S, Loos M. 1985. Effect of EDTA and citrate on the functional activity of the first component of complement, $\mathrm{C} 1$, and the $\mathrm{Clq}$ subcomponent. Immunobiology 170:123-132. 